\title{
1000 at 1000: an indentation toughness method
}

\author{
Robert F. Cook ${ }^{1, *}$ (1) \\ ${ }^{1}$ Winterville, USA
}

Published online:

13 July 2020

(C) Springer Science+Business

Media, LLC, part of Springer

Nature 2020

This editorial is part of the series "1000 at 1000" highlighting the most highly cited publications in the Journal of Materials Science as part of the journal's celebration of 1000 issues. In this issue: "Evaluation of $K_{\text {Ic }}$ of brittle solids by the indentation method with low crack-to-indent ratios," K. Niihara, R. Morena, D.P.H. Hasselman, [1] from the Virginia Polytechnic Institute and State University, Blacksburg, Virginia, USA.

In the 1980s, a technological drive to develop advanced structural ceramics was extremely active. The activity was based on the intrinsic advantages offered by ceramic materials, such as chemical and thermal stability, surface wear resistance, and bulk stiffness, along with dielectric and optical properties not found in metals. Structural (i.e., load bearing) components based on ceramic materials such as $\mathrm{Al}_{2} \mathrm{O}_{3}, \mathrm{ZrO}_{2}, \mathrm{SiO}_{2}, \mathrm{SiC}$, and $\mathrm{Si}_{3} \mathrm{~N}_{4}$ were developed and attention was naturally focused on optimizing the mechanical performance of such components, primarily by increasing ceramic strength. The strength of most ceramic materials at room temperature is controlled by brittle fracture: the ceramic sustains an applied stress elastically until a critical flaw becomes unstable at the maximum sustainable stress-the strength-and a crack propagates through the material leading to near instantaneous component failure. Many development efforts were therefore directed towards controlling processing of structural ceramics to minimize flaw size, thereby enhancing strength. The major effort in structural ceramic development, however, was directed towards optimizing processing to generate ceramic microstructures with increased resistance to crack propagation-increased toughness-thereby increasing strength. Hence, there was a need for methods to evaluate ceramic toughness and the development of such a method, using a commonly observed range of indentation crack lengths, was the focus of a much cited Letter by Niihara, Morena, and Hasselman [1].

Measurement of ceramic toughness using conventional methods can be an exacting task. Large

Address correspondence to E-mail: robertfcook@mac.com 
centimeter-scale specimens must be machined to prescribed geometries, such as the double cantilever beam, into which carefully-formed, long, straight cracks must be introduced. The specimens are then broken under controlled conditions, usually in a large, universal testing machine, and material toughness determined from the failure load characterizing instability of the crack in the specimen. Such measurements require specialized expertise and are time- and material-intensive. In contrast, measurement of a stable indentation crack appears to be a simple experimental task that is broadly implementable, rapid, and requires little material with minimal specimen preparation. A ceramic toughness determination method based on indentation crack length measurement thus has great appeal.

In a typical indentation fracture experiment, a foursided Vickers diamond pyramid is first loaded onto a ceramic surface by a gravity-loaded indenter. On removal of the load, a square residual contact impression remains, along with surface traces of cracks emanating from the impression corners, Fig. 1a. A localized, near-hemispherical zone of plastic deformation, mostly in the form of compressed shear faults, exists beneath the contact impression. The sub-surface geometry of the cracks, especially in opaque ceramics, is usually unknown but falls between the half-penny geometry, Fig. 1b, typified by soda-lime silicate glass at large indentation loads, and the radial geometry, Fig. 1c, typified by WC-Co composites. The localized plastic deformation zone generates a residual stress field in the surrounding elastically deformed matrix. The residual field initiates and stabilizes the cracks, which are typically 10 to $100 \mu \mathrm{m}$ in length and amenable to optical microscopy. Qualitative assessment of a material's tendency to deform and fracture is thus made easily and quickly, hence the popularity of indentation techniques. Quantitative estimation of material toughness by indentation crack length measurement, however, requires knowledge of the residual stress field and the crack geometry.

Niihara et al. [1] developed a quantitative indentation toughness method that largely assumed commonly accepted residual stress field descriptions but which implemented a new toughness analysis for small cracks. Specifically, Niihara et al. asserted that observations of "short" surface traces of indentation cracks implied sub-surface radial geometries, which in turn implied that a new or revised indentation toughness analysis was required in preference to the mainstream analyses based on the half-penny crack geometry. In quantitative terms, the Niihara et al. method is described briefly as follows. The residual diagonal of the square Vickers contact impression is $a$, Fig. 1. If the peak contact load forming the impression is $P$, the mean contact pressure or hardness, $H$, is thus

$H=P / 2 a^{2}$.

For most materials $H$ is found to be invariant (and related to the yield stress in metals [2]). The characteristic dimension of the hemispherical plastic deformation zone is $b$, Fig. 1 , such that the characteristic dilatational strain in the zone is $a^{3} / b^{3}$ and the characteristic pressure exerted by the zone on the surrounding matrix is thus $E a^{3} / b^{3}$, where $E$ is the Young's modulus of the material. In indentation halfpenny crack analyses, the characteristic projected area of the zone is recognized as $\sim b^{2}$ such that the pressure generated in the zone is manifest as an outward force of magnitude $F \sim\left(E a^{3} / b^{3}\right) b^{2}$ acting over the zone boundary at the center of the crack. Elastic-plastic analysis of an expanding spherical cavity shows that $b / a \sim(E / H)^{1 / 2}$ and hence, using

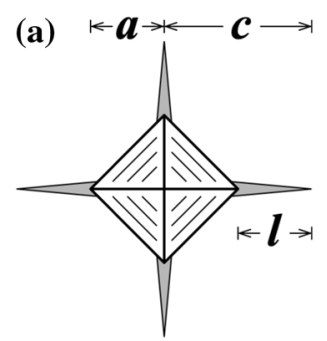

(b)

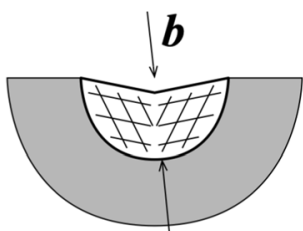

(c)

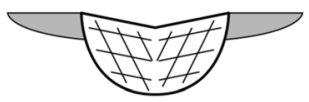

Figure 1 Schematic diagrams of Vickers indentation flaw. a Top view. $\mathbf{b}$ Side view of half-penny crack. c Side view of radial cracks. Dimensions are common to all views: $a$, impression semi- diagonal; $b$, plastic zone size; $c$, crack length measured from impression center; $l=c-a$, crack length measured from impression corner. 
this variation and the hardness relation above, the localized residual force wedging open the half-penny crack is related to the indentation load by $F \sim(E / H)^{1 / 2} P$. The stress-intensity factor (SIF), $K$, for a penny crack in a continuum loaded by a center force is $K=F /(\pi c)^{3 / 2}$, and hence the SIF for an indentation half-penny crack, Fig. 1b, is given by

$K_{\mathrm{hp}}=\xi(E / H)^{1 / 2} P / c^{3 / 2}$,

where the scalar parameter $\xi$ characterizes the geometrical details. Eliminating $P$ between Eqs. (1) and (2) and imposing the equilibrium condition $K_{\mathrm{hp}}=T$, where $T$ is the toughness (termed $K_{\mathrm{Ic}}$ in [1]), provides a relation including material and indention parameters that is the physical basis for analysis:

$T /(E H a)^{1 / 2}=\xi(a / c)^{3 / 2}$.

Niihara et al. began development of their revised methodology by assuming arithmetic modifications to Eq. (3):

$T /(E H a)^{1 / 2}=f_{1}(E / H) f_{2}(a / c)$,

where $f_{1}(E / H)$ and $f_{2}(a / c)$ were scalar empirical fitting functions based on selected data. Niihara et al. then noted that a prior set of indentation crack observations on WC-Co with small $c / a$ ratios were not well-described by the modified Eq. (4) and that indentation cracks in WC-Co were typically of the radial geometry. Niihara et al. then surmised that the deviation of the WC-Co data from Eq. (4) was due to the cracks exhibiting radial geometry and not the half-penny geometry on which Eqs. (3) and (4) were based. Niihara et al. then further surmised that small $c$ / $a$ ratios, less than about 1.5 to 2 , were a pervasive indication of radial geometry, and that therefore a new indentation toughness analysis was required for small cracks, described as exhibiting "low crack-toindent ratios" [1]. Niihara et al. then pointed out that such small indentation cracks are more appropriately described by crack length, $l$, measured from the impression corner, rather than $c$, measured from the impression center, Fig. 1a,

$l=c-a$.

Niihara et al. then modified Eq. (4) using the definition of Eq. (5) to arrive at

$T /(E H a)^{1 / 2}=A f_{1}(E / H)(a / l)^{1 / 2}$,

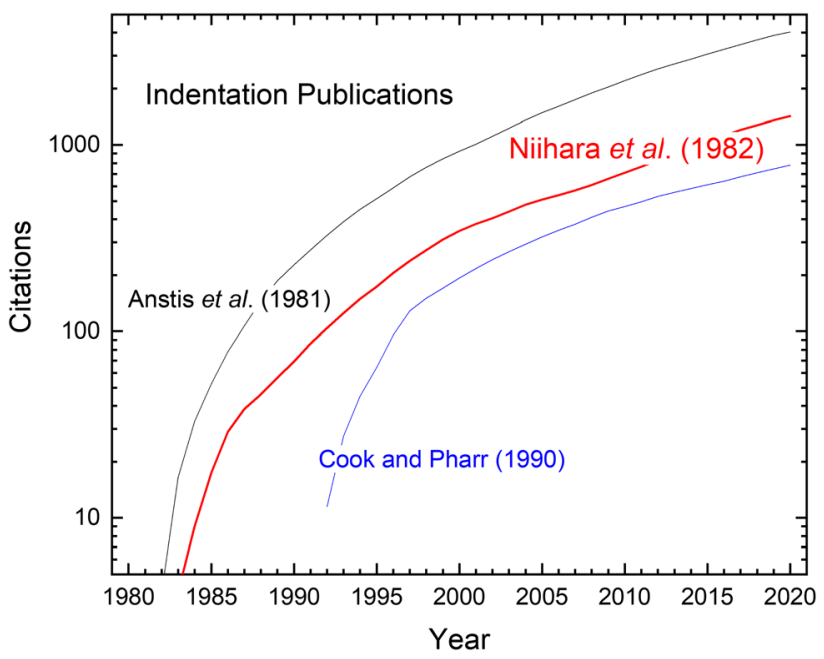

Figure 2 Cumulative yearly citations for the Niihara et al. [1] publication and two other publications focused on indentation fracture [3, 6]. Note semi-logarithmic scale; the interest in indentation techniques continues to increase. (Data compiled from Scopus courtesy M.L. Oyen.)

where $A$ was an adjustable fitting parameter. Equation (6) described the set of WC-Co data and a few other published measurements.

Equation (6) has been used frequently for toughness estimation using indentations and the Letter [1] containing the equation has been well-cited $(\approx 1500)$, Fig. 2. The basis and context of [1], however, has not been examined as frequently. Prior to the publication of [1], Anstis et al. [3] had published an extensive experimental evaluation of indentation toughness measurements. The Anstis et al. work is much more highly cited than the Niihara et al. work, Fig. 2, probably because the Anstis et al. work has a clear physical basis encapsulated in Eq. (2), contains extensive experimental measurements supporting Eq. (3) and a calibrated value of $\xi$, and, particularly, is more easily implemented numerically. Almost immediately after the publication of [1], Lankford [4] pointed out that the correlation noted by Niihara et al. to support Eq. (6) was an artifact of the plotting scheme selected and that, in fact, the data used by Niihara et al. and others could all be described by Eq. (3). In addition, in support of Eq. (3), Lankford provided a clear physical explanation why pairs of radial cracks behave as a single half-penny crack. Soon after, Laugier [5] provided a fracture mechanics analysis showing that the SIF for radial cracks, $K_{\mathrm{r}}$, was a weakly perturbed modification of that for halfpenny cracks, 
$K_{\mathrm{r}}=\xi_{\mathrm{r}}(a / l)^{1 / 2} K_{\mathrm{hp}}$

where $\xi_{\mathrm{r}}$ is geometrical correction factor of order unity. For small variations in relative crack length $(l / a)=(c / a)-1$, and hence small variations in indentation load, radial cracks are thus well-described by Eq. (3), consistent with observations of Anstis et al. and Lankford. Further, Laugier [5] showed that for such small variations, the equilibrium crack length characterized by $l$ should vary linearly with indentation load, $l \sim P$, consistent with the many prior observations of indentation cracks in WC-Co and implied by Niihara et al., Eq. (6). Somewhat later, in an extensive study focused on direct observation of indentation cracking, the present author in Cook and Pharr [6] noted that although indentation radial crack formation was much more prevalent in ceramics and glasses than previously thought, the indentation initiation loads and relative crack sizes were not restricted to the domain of small cracks. An historical overview, including [1, 4, 5], in the same work [6], makes clear that indentation crack surface trace lengths are a very weak indication of sub-surface crack geometry and hence a weak basis for analysis.

Although works contrary to the methodology suggested by Niihara et al. [1] are analytically and experimentally extremely well-based, not many are as well-cited. Citations to the works by Lankford [4] and Laugier [5] are far fewer $(\approx 250)$ as are (to a lesser extent) those to the work of Cook and Pharr [6] $(\approx 1000)$, Fig. 2. A partial explanation lies in the large number of citations $(\approx 5000)$ to Anstis et al. [3]. In both [1] and [3], simple, direct formulae are given for calculation of the quantity of interest, the toughness, using an apparently simple technique, indentation crack length measurement. The major explanation for the large number of citations to Niihara et al., however, probably lies in the instrumentation most commonly used to generate indentation cracks. Compact, small-load Vickers indenters with integrated optical microscopes became widely available in the 1980s, primarily intended for hardness testing of metals. The Vickers probes obviated the need for the large loads required in traditional spherical probe (e.g., Brinell) hardness testing [2] and such indenters thus often had a peak load of $10 \mathrm{~N}$. For a metal with hardness $H=1 \mathrm{GPa}$, this load is more than adequate to cause plastic deformation on contact and results in an impression dimension $a \approx 70 \mu \mathrm{m}$, Eq. (1), which is easily measured optically. For ceramics, however, this load is very close to the lower bound threshold for Vickers indentation cracking [7] and thus generates cracks with small surface trace/impression dimension ratios, $c / a \approx 1.5$, that are reduced further by the use of smaller loads and the ensuing difficulty of optical measurements. Hence, use of compact indenters, typically operated at a single, small, indentation load, generated relatively short cracks, for which the appropriate toughness analysis appeared to be the frequently cited [1].

Extensive analytical and experimental studies [3-7] suggest that the analysis and arguments forwarded in [1] might not be completely correct. However, from an engineering perspective, a recent overview [7] suggests that given the uncertainty in material properties and indentation geometry, the relative accuracy of indentation toughness evaluation methods is probably $\pm 50 \%$. Hence, any errors introduced by the use of [1] are probably well within the limits of the technique. More important from an engineering perspective is that Fig. 2 shows citations to [1, 3], and [6] continuing to increase in a near exponential manner. The development and application of structural ceramics is clearly ongoing, e.g., handheld device screens. Materials science based assessments of the quantitative linkages between flaws, microstructure, toughness, and strength are clearly required to advance the materials engineering goal of increasing load-bearing capacity in application. Hence, the evaluation of structural ceramics properties by indentation remains of great interest. Ideas regarding crack initiation [6], crack geometry [1], and indentation load dependence [3,7] should be introduced, questioned, and explored in order to better understand brittle fracture strength and more rapidly develop advanced structural ceramics.

\section{References}

[1] Niihara K, Morena R, Hasselman DPH (1982) Evaluation of $K_{\text {Ic }}$ of brittle solids by the indentation method with low crackto-indent ratios. J Mater Sci Lett 1:13-16

[2] Tabor D (1951) The hardness of metals. Clarendon Press, Oxford

[3] Anstis GR, Chantikul P, Lawn BR, Marshall DB (1981) A critical evaluation of indentation techniques for measuring 
fracture toughness: I, direct crack measurements. J Am Ceram Soc 64:533-538

[4] Lankford J (1982) Indentation microfracture in the Palmqvist crack regime: Implications for fracture toughness evaluation by the indentation method. J Mater Sci Lett 1:493-495

[5] Laugier MT (1985) Palmqvist crack extension and the centerloaded penny crack analogy. J Am Ceram Soc 68:C51-C52
[6] Cook RF, Pharr GM (1990) Direct observation and analysis of indentation cracking in glasses and ceramics. J Am Ceram Soc 73:787-817

[7] Cook RF (2020) A critical evaluation of indentation crack lengths in air. J Am Ceram Soc 103:2278-2295

Publisher's Note Springer Nature remains neutral with regard to jurisdictional claims in published maps and institutional affiliations. 